\title{
Information processing in visual search: A continuous flow conception and experimental results
}

\author{
CHARLES W. ERIKSEN and DEREK W. SCHULTZ \\ University of Illinois, Champaign-Urbana, Illinois 61820
}

\begin{abstract}
This paper reexamines the visual search process, and visual information processing more generally, from a perspective of the continuous flow of information and responses through the visual system. The results from three experiments are reported which support the continuous flow conception: Information accumulates gradually in the visual system, with concurrent priming of responses. The first two experiments investigated the processing of display stimuli which varied in size and figure-ground contrast in a nonsearch task, and provided evidence confirming a continuous flow model. Experiment 3 employed an asynchronous onset of target and noise and provided convergent evidence of the accumulative nature of information and response priming in visual processing.
\end{abstract}

In one form of visual search, subjects are required to recognize or detect a target letter or form embedded in a display of noise consisting of other letters or forms. Not surprisingly, both speed and accuracy on this task have been found to depend upon the number of noise elements present in the display and the similarity of these noise elements to the target (Eriksen \& Spencer, 1969; Estes \& Wessel, 1966; Townsend, Taylor, \& Brown, 1971; Wolford, Wessel, \& Estes, 1968). But while this result seems reasonable, the mechanisms or means by which noise exerts its effects are not readily apparent. Attempts to elucidate the role of noise have given rise to a number of models, some of which have located the effects at early perceptual stages of processing (Bjork \& Murray, 1977; Estes, 1974) and others which have emphasized the decisional stage (Gardner, 1973; Kinchla, 1974; Shiffrin \& Geisler, 1973). Although some of the models are quite detailed and elegant, none encompass all the variables and factors that have been shown to enter into visual search.

\section{Decision Level Effects}

Eriksen and Spencer (1969) have shown that, with respect to detection or recognition, increasing the number of noise elements increases the opportunities for confusions and false alarms. In energy-limited displays, there is a finite probability on a trial that any

This research was supported by Public Health Service Research Career program Award K6-MH-22014 to the first author and by U.S. Public Health Service Research Grant MH-01206. Requests for reprints should be directed to Dr. Charles W. Eriksen, Department of Psychology, University of Illinois at UrbanaChampaign, Champaign, Illinois 61820. The authors wish to thank Mr. Barry Schaff and Mrs. Connie Daye for assistance in data collection. given noise element may reach the subject's criterion for detection or recognition. Thus, increasing the number of noise elements increases the probability that at least one noise element will lead to a false detection. Estes has extended this account of the role of noise in visual search (Estes, 1972, 1974).

The increase in search time that occurs with an increase in the number of noise elements also can be attributed, in part at least, to the search process itself. Although, in most search experiments, the duration of the display is too brief to permit changes in eye fixation, the concept of an internal scan mechanism that serially searches a sensory register is still viable. This scanning concept recently has been invoked by Bryden (1960), Mewhort (1974), Shaw and Weigel (1973), Von Wright $(1968,1970)$, and others. If a central scanning operation is posited, then, as the total number of items in a display increases, detection or recognition time would increase due to the increase in the number of items to be scanned.

It has been found, however, that the effects of noise stimuli are more pervasive than can be accounted for in terms of target-noise confusion or the time required for a central scanning process. The possibility of an internal scan or of target-noise positional confusion is eliminated if the target always occurs in a known location so that a search operation is not required. Eriksen and his associates (Colegate, Hoffman, \& Eriksen, 1973; Eriksen \& Collins, 1969; Eriksen \& Hoffman, 1972, 1973) have employed a technique involving a circular arrangement of letters centered upon a fixation point. The location of the target letter is precued by a prominent black indicator as much as 300 to 400 msec before display onset. Both accuracy and reaction time for identification of the target letter have been employed as dependent variables (Eriksen 
\& Hoffman, 1972; Eriksen \& Rohrbaugh, 1970). These experiments have shown that even when the target location is known to the subject beforehand and no search is required, recognition accuracy and decision time are still influenced by the number of noise elements in the display and target-noise similarity.

One may question whether the precueing by a prominent bar marker several hundred milliseconds before the display onset is sufficient to provide the subject with unequivocal location information. Estes, Allmeyer, and Reder (1976) found that positional uncertainty exists in linear displays, and Strangert and Brännström (1975) and Wolford and Hollingsworth (1974) have shown that spatial proximity of target and noise impairs target recognition and decision time. If positional uncertainty as to the exact location of the target remained, then it would still be necessary for the subject to scan elements in the vicinity of the target and the possibility of target-noise confusion would still exist.

However, there is considerable evidence against such a possibility. First, Eriksen and Rohrbaugh (1970) found that increasing the number of noise elements in the circular displays decreased recognition accuracy even when the spacing between target and adjacent noise was held constant. Second, Colegate, Hoffman, and Eriksen (1973) varied the time interval by which the indicator preceded the display over a range of $500 \mathrm{msec}$ and found that, although decision time became asymptotic when the indicator preceded the display by 250 to $300 \mathrm{msec}$, displays with differing numbers of noise letters approached different asymptotes.

Even more convincing that the effects of noise are not attributable to insufficient time for the subject to determine the target location are the results of experiments by Eriksen and Eriksen (1974) and by Eriksen, Hamlin, and Daye (1973). These experiments employed a technique in which the subject knew that the target would always appear $.25^{\circ}$ above the fixation point. He was further instructed to ignore any other stimuli that might appear in the visual field. In both studies, reaction time to a target letter was increased significantly if noise letters flanking the target appeared simultaneously in the visual field. In the second study, these effects were found to persist even when the noise elements were separated from the target by a horizontal distance of $1^{\circ}$ of visual angle. More recently, O'Hara (1977) found noise effects upon target decision time when target and noise separation was $1.8^{\circ}$ of visual angle, and Gatti and Egeth (1978) have found effects at $5^{\circ}$ separation. It is difficult to argue that, at these separations in the visual field, the subject was still uncertain about the target location and had to scan some noise elements as well as the target.

\section{Early Perceptual Leve! Effects}

The above evidence is quite clear in indicating that the effects of noise cannot be attributed solely to a decision or central scanning stage in a visual information processing. Banks and Prinzmetal (1976) have emphasized the early perceptual stage in the separation of target and noise and have proposed a configurational hypothesis of visual detection. Their hypothesis draws heavily on the Gestalt principles of perceptual grouping and organization. The structuring or grouping of visual stimuli occurs at an early stage in perception, somewhat comparable to Kahneman's (1973) unit formation or Neisser's (1967) preattention stage. The time required for detection or recognition of the target depends upon the degree to which the target merges with, or stands out from, the noise characters in this early structuring. Banks and Prinzmetal found that when a target element was embedded in noise characters arranged in such a way as to form a "good figure," the time needed to detect the target was longer than when the noise characters were arranged so as to destroy the "good figure." In further work, Prinzmetal and Banks (1977) found that the Gestalt principle of good continuation could predict the ease with which a target was detected in a visual display.

Estes (1974) also has suggested that some of the interference of noise letters occurs on the input or early perceptual side of the processing chain. In his interactive channels model, Estes (1974) has theorized that the inputs from noise letters have an inhibitory effect upon the input channels from the target letter. In this conception, the task of the processing system is to synthesize the percept of a letter on the basis of information coming to feature detectors from a local region in the visual field. The magnitude of the inhibitory effect depends upon the degree to which the set of feature detectors activated by the noise letters overlaps the set of detectors activated by any of the letters of the target set. Thus, the inhibitory effect of noise letters depends in part upon the overlap of noise features with the target set and upon the separation of the noise elements from the target in the visual field.

\section{Response Level Effects}

So far we have considered attempts to localize the effects of noise at the input and at central stages of information processing. What remains for consideration is the output or response side of the processing sequence. An experiment by Eriksen and Eriksen (1974) offers strong evidence that noise letters in a display can produce pronounced effects at the response level. In their experiment, four target letters were divided into two sets. One set required a lever movement to the left, the other one, to the right. A single target letter was presented to the subject at a 
known location immediately above the fixation point. On some trials, the target letter was flanked by letters of the same target set (compatible), on other trials by letters of the other target set (incompatible) or by neutral letters not associated with an experimentally defined response. These neutral letters shared different amounts of featural overlap with the target set letters.

When the target was flanked by letters of the opposite response set, reaction time to the target letter was markedly increased relative to when the noise letters were from the same response set as the target. The neutral noise letters produced intermediate effects upon reaction time, with the magnitude of their effect depending upon the number of features they shared with response-compatible or -incompatible letters. Neutral letters having a high feature overlap with the incompatible response set produced longer reaction times than neutral letters whose features were more similar to the compatible response set. In all cases, the effects upon reaction time were maximal when the noise letters occupied spatial positions close to the target letter with the effects decreasing (but not disappearing) as letters were moved out to a degree of angle separation. Compatible results have been obtained by Keren, O'Hara, and Skelton (1977) and by O'Hara (1977), employing a same-different discrimination.

There are two important conclusions to be drawn from the Eriksen and Eriksen study. First, subjects cannot restrict their attention to process only a single letter, even when the location of this letter is clearly designated and known beforehand. In other words, the minimal size of the attentional channel encompasses at least two letters. Whether the target and noise letters are processed in strict parallel fashion is essentially moot, but the evidence strongly suggests that multielement processing is at least an overlapping process. The processing system seems incapable of structuring, on the basis of instructions or set, the order in which the available stimuli are processed to the response stage.

The second conclusion is that the noise letter (or letters) is processed along with the target to the point of incipient response activation. This follows from the salient finding that response-incompatible noise letters produced considerably greater impairment in reaction time than did response-compatible or neutral letters. In fact, when target and noise separation was great enough to eliminate contour interference (Flom, Weymouth, \& Kahneman, 1963), responsecompatible noise had little or no effect. Since the effect of a noise letter could be manipulated by its experimentally defined response compatibility, the interfering effects cannot be attributed solely to Gestalt organizations, decision processes, or feature interference.
These conclusions are not new. The extensive research on the Stroop effect has yielded similar conclusions as to nonselective multielement processing and response interference (Dyer, 1973; Neill, 1978; Proctor, 1978). What is novel about the Eriksen and Eriksen findings is the clear demonstration that response competition is a potent variable in the visual search task.

The results of these response interference experiments rule out visual search models employing discrete successive stages of the form: input $\rightarrow$ central decision process $\rightarrow$ response activation. The experiments show that an appreciable component of noise interference is at the response level. This would not happen if responses were activated only after a decision had been made. Amount of noise and its similarity to the target can affect the speed with which information becomes available to a decision process and also the latency of the decision, but once the decision is reached and a response activated, response compatibility of the noise would not have an effect. The decision process would effectively block the access of competing noise stimuli to the response system.

\section{Sensory System Effects}

Not only have current visual search models not dealt with the response interference effects in the search task, but they have tended to ignore some of the characteristics of the visual sensory system that are highly pertinent to search. The role of visual acuity in distinguishing targets from noise is selfevident. If the target differs from the accompanying noise only in terms of minute featural detail, we would expect errors to be numerous and search time increased. Experimentation is hardly required to demonstrate this obvious result. But the variables underlying visual acuity are often overlooked and confounded in visual search tasks.

Visual acuity is defined as the minimal separation in terms of degrees of arc that can be resolved by the eye under a specified set of conditions. These conditions include retinal locus, figure-ground contrast, viewing time, and luminance. Acuity is best in the foveal center but decreases rapidly as stimulation is moved peripherally. Not surprisingly, recognition latency also varies with foveal location. Eriksen and Schultz (1977) have shown that reaction time to identify a letter increases about $100 \mathrm{msec}$ as the letter is moved from fovea center to $3^{\circ}$ along the horizontal meridian. Similar findings have been reported by Lefton and Haber (1974). It is obvious that models of visual information processing that assume simultaneous availability to central processors of all items in displays are inappropriate.

Reductions in acuity due to retinal locus or other variables such as figure-ground contrast or limited viewing time can, within limits, be compensated by an 
increase in energy (luminance). This is particularly pertinent since it has been well established that the visual sensory system integrates energy over temporal intervals in excess of $100 \mathrm{msec}$ (cf. Ganz, 1975). In fact, the work of Kahneman and his associates (Kahneman \& Norman, 1964; Kahneman, Norman, \& Kubovy, 1967) has indicated that for form perception, timeintensity reciprocity may be in excess of $200 \mathrm{msec}$. The improvement in acuity with increased viewing time or exposure duration of the stimulus can in most instances be attributed to increased energy summation over the longer temporal interval.

Eriksen and Schultz (1978) have pointed out that the summation of energy in the visual system implies that the accumulation of information about a stimulus in a sensory register or the percept is a temporally distributed process. They draw the analogy to a photographic film immersed in developer. Information becomes available first as gross figure-ground differentiations. As the developer acts upon the film, increasingly finer details become discernible. If this analogy fits the visual system, target discriminations that have a high acuity requirement would have longer latencies than those with a lower acuity requirement. Discrimination would be delayed until the visual system summed sufficient energy to compensate for the differences in acuity requirement. Similarly, other things being equal, stimuli located several degrees from fovea center would have to await more energy summation before they would become discriminable. As we have seen, the results of Eriksen and Schultz (1977) and Lefton and Haber (1974) support this latter inference.

\section{A Continuous Flow Conception}

Visual information developing over time (albeit on the order of 100 to $200 \mathrm{msec}$ ) and the response competition effects discussed earlier suggest a perspective of the search task that we designate as a continuous flow conception. In this conception, information about stimuli accumulates gradually in the visual system, and as it accumulates, responses are concurrently primed or partially activated. We conceive of several processes or levels comprising the events from stimulation to response activation. With the onset of stimulation, input channels begin to feed a continuous output to feature detectors which, in turn, continuously feed to form units. The output from the form units is a priming or activation flow to the response system. The output from each process becomes increasingly more detailed or exact over time as energy is integrated in the visual sense organ. The effect at the response level, with this continuous flow, is an initial priming of a wide range of responses. But as the processing at the lower levels proceeds in time, the priming flow becomes increasingly restricted to fewer and fewer responses, namely, those that are still viable alternatives in terms of the increasingly more exact or complete output of the lower processes.

A main feature of this conception is that there is not a discrete decision stage between input and responses. As the percept develops, responses receiving priming are held in check by an inhibitory process. When the priming for an internal recognition response or an overt response reaches the evocation threshold, the inhibition is removed and the response occurs. The evocation threshold, in turn, is capable of a limited range of variation. Learning and practice would be expected to produce differential evocation thresholds among responses. But the effects of instructions, set, or expectancy are probably more parsimoniously conceived as an initial priming of the expected responses. Instructions, or set, as well as other variables, such as target/noise confusability and payoff schedules, act to differentially prime certain responses over other responses. As a consequence, a preprimed response would reach evocation threshold at a point in time where the percept is less fully developed.

Noise stimuli in visual search produce a response competition effect when the responses they prime are incompatible with the target response. An important limitation in the human as an information processor lies on the response side. We cannot simultaneously say the letter $\mathrm{A}$ and the letter $\mathrm{B}$, or move a response switch both right and left at the same time. Introspection suggests that the internal recognition responses also are serial in nature. If the recognition process by which we name or encode letters and objects into a short-term memory is serial in nature, it too may be subject to response competition effects. Although Eriksen and Colegate (1971) have presented evidence indicating that encoding letters from a visual display is a serial process, whether or not response competition occurs in terms of an internal recognition response is still an empirical question. One of the purposes of Experiment 3, described below, was to furnish evidence on this issue.

The amount of competition between incompatible responses is conceived as a function of the amount of priming each response receives. A target response will be inhibited to a degree determined by (a) the number of alternative incompatible responses that are being primed or activated, and (b) the amount of priming these competing responses receive.

The amount or level of priming that a competing response will receive is, in turn, determined by the amount of featural overlap it has with the target. If the target and noise letters are quite similar (high feature overlap), the flow of priming to the competing noise letters will continue longer as the percept develops, since it will not be until the last stages of percept development that the distinguishing features of the 
target will be available as a basis for a discriminated response.

We do not conceive of the continuous flow model as a complete description of visual search. Rather, it is an outline within which experimentation can suggest the form that more detailed exposition should take. The continuous flow perspective suggests that reaction time to a target in a visual display can be decreased by making the target (a) larger, (b) brighter, (c) higher in contrast, or (d) by placing it in a more sensitive foveal location than the response competitive noise. All of these manipulations would insure that target information reaches criterion while the priming of incompatible responses is at a lower level. Alternatively, the interference from incompatible noise will be increased with a concomitant increase in the reaction time to target if the noise elements are relatively enhanced by the above manipulations. In this case, their responses will receive relatively more priming before the target criterion is reached.

\section{EXPERIMENT 1}

In this experiment, we manipulated the relative processing time of target and noise letters, and thus the amount of response competition, by varying the size and the figure-ground contrast of the target. A nonsearch paradigm was employed in which the target letter $(\mathrm{A}$ or $\mathrm{H})$ always appeared immediately above the fixation point. In the response-compatible noise condition, the target was flanked by four replications of itself (e.g., A A A A A). In the incompatible condition, the target was flanked. by four instances of the other target letter (e.g., H H A H H). There were five target conditions: In the baseline condition, the target and the noise letters were black and the same size, while in the size condition, the target was twice as large as the noise letters. In the contrast condition, the target had a much lower contrast with the ground than did the noise letters, and in the size/contrast condition, the target was twice as large as the noise elements but of lower contrast. Finally, a no-noise control was run in which the target appeared alone in an otherwise empty field. Under all conditions, the subject was instructed to respond with a right or left lever movement appropriate to the target.

The manipulation of target size followed from the results of a previous experiment (Schultz \& Eriksen, 1978), where RT to single target letters was found to decrease significantly when their size was increased from $.125^{\circ}$ to $.5^{\circ}$ of visual angle. Figure-ground contrast was manipulated in the following way. A bright yellow color was chosen for the target. This yellow had a low contrast with the white background of the stimulus card but at the same time made the target "distinctive." Pilot investigation had shown that when subjects were required to name colors as quickly as possible, the yellow was named more rapidly than red, blue, or black, but forms presented in this yellow were recognized more slowly due to the lower contrast.

A further manipulation involved blocked and nonblocked trials. In blocked trials, the noise remained the same for each of 24 trials in the block (e.g., all As), but the target varied randomly between $A$ and $H$. For the nonblocked trials, the noise varied randomly from trial to trial, as did the target letter. Subjects were informed of the experimental condition before the beginning of a block of trials.

The continuous flow model makes several predictions as to the outcome of this experiment. Relative to the baseline condition, RT will be faster under the size condition and slower under the contrast condition across the experiment. Target latencies under the size/contrast condition will be intermediate. When the target is larger than the noise letters (size condition), less energy summation time in the visual system will be required to resolve details necessary for its recognition; conversely, in the contrast condition, the lower figure-ground contrast of the target will require longer energy summation. Intermediate latencies are expected for the size/contrast condition, since the effect of increasing target size will be offset, at least in part, by its lower contrast.

The noise-compatibility variable is predicted to have a pronounced effect. In the compatible condition - where the noise consists of replications of the target -no competing responses are activated to inhibit target reaction time (RT). However, small differences in RTs under the different target conditions are to be expected due to differences in the processing time of the target as a function of its size or contrast.

In the incompatible condition, where the noise elements are the other target, maximum response competition is activated and proceeds to the final levermovement response. Further, the presence of strong competing responses will enhance differences between the target conditions. Target RT will be affected least under the size condition, where the faster processing time for the large target will result in its recognition while the level of priming in the competing response is still at a lower level. The opposite result will occur for the contrast condition, where the slower processing time for the target allows the competing noise response to achieve a higher level of priming and thus produce greater interference to the target response.

The use of blocked and nonblocked trials was primarily exploratory. We wished to determine the extent to which noise effects could be attenuated if the subject knew their specific identity before a trial. If the subject knew that the target, $A$ or $H$, would appear on a background of Hs for the next series of trials, he might be able to adopt a more efficient set of cues for target identification. He could use an "identity detector" (Bamber, 1972) to discriminate 
H H H H H from $\mathrm{H} \mathrm{H} \mathrm{A} \mathrm{H} \mathrm{H}$ rather than identifying the $\mathrm{A}$ or $\mathrm{H}$. He might also be able to inhibit, in some fashion, the competitive effects of noise if the nature of the noise was known beforehand. The results of the experiment could provide information on these two possibilities in the following manner. If the subject were able to use the information to set a more efficient criterion, then performance with both compatible and incompatible noise should be faster under the blocked trials condition than under the nonblocked condition. On the other hand, if the subject were able to use the information only to minimize the competitive effects of incompatible noise, an interaction between trial blocking and noise compatibility would occur. Performance for compatible noise would be comparable under both blocked and nonblocked conditions, but would be faster for incompatible noise under blocked trials than under nonblocked trials.

The no-noise control was employed to ascertain the extent of basement effects, particularly in the compatible noise condition. A failure to obtain differences between target conditions in the compatible noise conditions, and between blocked and nonblocked trials, could be due to a basement effect where RTs were so fast that differences between conditions could not be detected. The no-noise condition served as a marker for this possible effect.

\section{Method}

Subjects. Eight volunteers, all male, were paid \$6 for serving in five sessions. Four of the subjects were patients at the Danville Veterans Administration Hospital in the Alcohol and Drug Rehabilitation Unit, but were not receiving medication that would be apt to affect their performance. One subject was an employee at the Danville VA Hospital, and the other three were students at the Danville Junior College. The subjects were selected on the basis of pretesting, wherein they maintained an error rate less than $15 \%$ for latencies under $500 \mathrm{msec}$.

Apparatus and Stimuli. Trials were conducted in two channels of a three-channel tachistoscope illuminated with Sylvania F4T5/ CWX bulbs. Viewing was binocular. The luminance of both fields was set at $11.24 \mathrm{~cd} / \mathrm{m}^{2}$ as measured by a Macbeth illuminometer. and stimulus duration was $1,000 \mathrm{msec}$. The fixation field remained on except when the stimulus field was illuminated. The subject's response was always a lever movement to the left or right, which stopped the Lafayette Model 54517 clock counter. The clock counter had been activated simultaneously with the onset of the stimulus display.

Twenty-four stimulus displays were constructed on white vinyl cards using Presstype Futura Bold 24- and 48-point black or yellow letters. The smaller letters subtended $.25^{\circ}$ in both width and height, and the larger letters $.50^{\circ}$ in both width and height. The displays were constructed so that the centered target letter always appeared $.25^{\circ}$ of angle above a black fixation " $x$," subtending $.25^{\circ}$ in both width and height. Size and color of the target letter were varied across all four possible combinations of 24- or 48-point size and black or yellow color (yellow targets had an approximate Munsell notation of $8 / 10$ ). Sixteen of the stimulus displays were constructed so that each of the eight possible targets could be horizontally and symmetrically flanked by four 24-point black noise elements. Noise elements were homogeneous within a display (either all As or all $\mathrm{Hs}$ ) and were symmetrically separated from the target and each other by $.25^{\circ}$ of angle intercontour distance.

Procedure. Each subject served in five sessions, the first of which was practice. Each session was conducted under one level of the four possible size/contrast combinations for the targets, and the subject was informed of the conditions at the outset of the session. A session consisted of 15 initial warm-up trials, and five blocks of 24 trials each. Two of these blocks were conducted under a mixed noise condition: 12 randomly chosen trials contained Hs for noise, and 12 contained As. In two other blocks, the particular noise element was held constant and the 24 trials contained either all As or all Hs as the noise. Finally, one block was run under a no-noise control wherein the target appeared alone. The target ( $A$ or $H$ ) was randomized across trials within each block, and block order was counterbalanced within subjects.

The subject was dark-adapted, and then shown 15 practice trials. He was instructed to focus clearly on the fixation cross and was warned by the experimenter's signal, "Ready," when the trial was about to begin. He was to respond by a horizontal hand lever movement in the direction appropriate to the target, and direction of lever movement was counterbalanced across subjects. The subject was always informed that the noise elements flanking the target should be ignored and that he would never be asked to recall or use them in any way. He was also reminded that the target would always appear in the same place: "just above where the ' $x$ ' has been." The sessions typically lasted $50 \mathrm{~min}$, and informal debriefing followed the final session.

\section{Results and Discussion}

The RT data were analyzed by a four-way ANOVA (noise compatibility, target conditions, trial blocking, subjects). All main effects were significant ( $p<.001$ ). In addition, noise compatibility interacted significantly with target conditions $(F=8.03, \mathrm{df}=3,21)$ and with trial blocking $(F=32.47, \mathrm{df}=1,7)$. These effects are seen in Figure 1, where mean latency is shown for target conditions in blocked and nonblocked trials as a function of noise compatibility. Results for the no-noise trials are plotted to the right of the figure.

Overall, target conditions were ordered in latency as predicted. Relative to the baseline condition, RTs were quicker when the target was larger than the surrounding noise and slower when the target was lower in contrast. The intermediate result for the size/ contrast condition was predicted on the basis of the larger size partially counteracting the effect of the re-

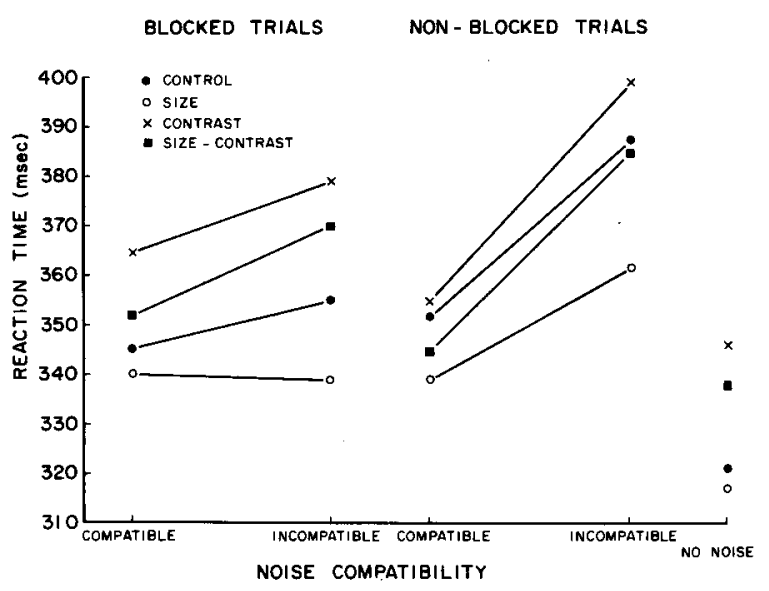

Figure 1. Mean latency for the target conditions under blocked and nonblocked trials as a function of noise compatibility. 
duced contrast. The same ordering of target conditions is observed under the no-noise control.

Also as predicted, noise compatibility had a pronounced effect upon target RT: latency under the noise-incompatible condition was longer than when the noise was compatible. The significant Noise Compatibility by Target interaction is consistent with the prediction that competing responses would enhance differences between the target conditions. The differences in target conditions are assumed to reflect differences in the summation time necessary to resolve critical detail in the visual system. For example, the longer summation time required for the low contrast target permits a higher level of priming of the competing response from the high-contrast noise. Conversely, with the larger target, the shorter summation time for the target results in a relatively lower level of priming of the competing response from the smaller noise elements.

The smaller differences among target conditions with compatible noise reflect the differences in summation time in the visual system due to variation in target size and contrast. This contention is supported by the observation that for both blocked and nonblocked trials under compatible noise, the differences among target conditions are on the same order of magnitude as those obtained for the no-noise control.

For all target conditions, blocked trials were significantly faster than nonblocked trials, and trial blocking interacted significantly with noise compatibility. Since trial blocking was an exploratory variable, no specific predictions were made as to its effect. Two possible ways that a subject could use the advance information of the noise letters before a trial were noted, however. One method would be to adopt a more efficient set of discriminatory cues. The other would be to minimize the competing effect of the noise by some differential inhibition of the noise response. The interaction obtained between trial blocking and noise compatibility supports the latter alternative. Had the subject found a set of more efficient discriminatory cues, differences between blocked and nonblocked trials under the compatible noise conditions should have resulted. However, examination of Figure 1 shows that performance for both blocked and nonblocked trials under compatible noise was comparable with the possible exception of the contrast condition.

Performance of target conditions under the compatible noise is appreciably slower than under the nonoise control. However, the ordering of the target conditions, and the magnitude of the differences between them, are comparable. A three-way ANOVA (target conditions, no-noise vs. compatible noise, and subjects) showed that the no-noise conditions were reliably faster than compatible noise conditions $(\mathrm{p}<$
$.01)$, as were also the differences between target conditions $(p<.05)$. The interaction did not approach significance.

The faster RTs obtained with the no-noise control can be attributed in part to the fact that these trials were run in blocks during which the subject knew that the target letter would appear alone without accompanying noise letters. Eriksen and Eriksen (1974) previously found that when no-noise trials were blocked as opposed to randomly intermixed with trials containing noise elements, the blocked trials had significantly faster reaction times. Eriksen and Eriksen noted that if subjects are processing target and noise letters simultaneously, accurate performance on the task requires that subjects employ an inhibitory mechanism to minimize erroneous responses. With two or more responses essentially simultaneously available, the subject might perform a "check" operation to make sure which response corresponded to the target location. When a subject knows that a block of trials will not include noise letters, this inhibitory process can be discarded, with a subsequent reduction of reaction time.

A comparison of RTs under different experimental conditions always raises the question of whether differences in speed may not be due to changes in the subject's criterion (speed-accuracy tradeoff). Criterion change does not appear to be a factor in the present data. Table 1 presents the mean errors committed under the different treatment conditions. While the experiment was not designed to be very sensitive to an error analysis, inspection of Table 1 shows that the error data have the same trends as the RTs. A product-moment correlation of mean RT and mean errors across the 20 treatment combinations yields a value of .653 .

\section{EXPERIMENT 2}

In the previous experiment, making the target letter larger than the accompanying noise letters was quite effective in reducing the interference of these noise letters when they were incompatible with the target. This effect had been predicted from the shorter summation time required to resolve the details of the

Table 1

Mean Percent Errors for the Different Treatment Conditions

\begin{tabular}{|c|c|c|c|c|c|}
\hline \multirow[b]{2}{*}{$\begin{array}{c}\text { Target } \\
\text { Conditions }\end{array}$} & \multirow[b]{2}{*}{$\begin{array}{c}\text { No } \\
\text { Noise }\end{array}$} & \multicolumn{2}{|c|}{$\begin{array}{c}\text { Compatible } \\
\text { Noise }\end{array}$} & \multicolumn{2}{|c|}{$\begin{array}{c}\text { Incompatible } \\
\text { Noise }\end{array}$} \\
\hline & & Blocked & $\begin{array}{c}\text { Non- } \\
\text { blocked }\end{array}$ & Blocked & $\begin{array}{c}\text { Non- } \\
\text { blocked }\end{array}$ \\
\hline Control & 6.7 & 4.1 & 3.1 & 9.3 & 15.1 \\
\hline Size & 4.6 & 4.1 & 2.6 & 13.0 & 15.0 \\
\hline Contrast & 3.5 & 3.1 & 3.2 & 12.5 & 16.7 \\
\hline Size/Contrast & 4.1 & 4.1 & 1.5 & 12.5 & 17.7 \\
\hline
\end{tabular}


larger target. As a result, there was less response priming for the more slowly processed incompatible noise letters at the point of target recognition and, therefore, less inhibition of the target response.

However, making the target larger than the noise also makes the target distinctive in ways not necessarily associated with visual summation time. For example, increasing target size renders the target location less ambiguous. While the contrast condition would also seem to satisfy these same characteristics of distinctiveness, a more appropriate control for any distinctiveness effects would be to reverse the size condition: make the target small and the noise letters large. If some distinctiveness variable, such as reduced ambiguity of target location, were responsible for the size-condition results in Experiment 1, then making the target half the size of the noise letters should also reduce the interference of incompatible noise. Conversely, the continuous flow model would predict that making the incompatible noise letters larger than the target letter would enhance their competitive effect, since the summation time in the visual system for identification would now be faster than for the smaller target, and the level of priming for the competing response would be concomitantly higher.

\section{Method}

The same experimental paradigm and equipment as in the previous experiment were employed. $A$ target letter $(A$ or $H)$ was flanked by four noise letters in the response-compatible (e.g., A A A A A) or response-incompatible ( $\mathrm{H} \mathrm{H} \mathrm{A} \mathrm{H} \mathrm{H)} \mathrm{condition.} \mathrm{All}$ trials where noise was presented with the target were blocked. Thus, the subject knew before every block of trials that the noise letters would always be As (or Hs) for all the trials in that block.

Three target conditions were studied. In Condition 1, the target and the accompanying noise letters were all $.25^{\circ}$ of visual angle in height (24-point Futura Bold). In Condition 2, the target letter remained $.25^{\circ}$ of angle while the noise was $.50^{\circ}$ (48-point). In Condition 3 , the target again was $.25^{\circ}$ but the noise was now $1.12^{\circ}$ of angle (96-point). Condition 1 was the same as the baseline condition of Experiment 1 and Condition 2 was the reverse of the targetnoise size relation of the size condition of the previous experiment. Condition 3 was run to ascertain a limit to the latency facilitation obtained by increasing the relative size differences between target and noise. At some point, the letter becomes so large that its critical details may fall on areas of less acuity in the retina and thus require longer processing time (Eriksen \& Schultz, 1977). Moreover, if the noise letters become too large, they are apt to be structured as ground against which the target will be perceived as figure, thus facilitating target processing (Banks \& Prinzmetal, 1976). From these considerations, it was expected that under Condition 3 the noise might be less effective than under Condition 2 .

Finally, no-noise trials (in which the target letter occurred alone in the visual field) were run under two conditions: In the blocked no-noise condition, the subject knew that the entire trial block would consist only of target letters. In the mixed no-noise condition, no-noise trials were intermixed randomly with the noise trials. From the discussion of Experiment 1, it was predicted that no-noise target latencies would be faster under the blocked condition.

Each subject participated in four sessions, the first of which was practice. Each of the three experimental sessions employed only one of the noise size levels. Each session consisted of three blocks of trials. In one block, the noise was always As and in the other block always Hs. In both of these blocks of trials, 24 no-noise trials were randomly interspersed with the noise trials for a total of $\mathbf{4 8}$ trials per block. The third block consisted only of no-noise trials. Block order and target conditions were counterbalanced both within and between subjects.

The subjects were nine volunteers. Three were patients at the Danville Veterans Administration Hospital, four were VA employees, and two were Danville Junior College students.

\section{Results and Discussion}

Data for the noise trials were analyzed in a threeway ANOVA (target conditions, noise compatibility, and subjects). The main effects for target conditions and noise compatibility were significant $(\mathrm{p}<.01)$, as was the interaction of these two variables $(\mathrm{p}<.01)$. The mean latencies for the three target conditions under the compatible and incompatible noise are shown in Figure 2.

Data under Condition 1, which was identical to the baseline condition of Experiment 1, replicate the results of that experiment. In the baseline condition of Experiment 1, incompatible noise produced an approximately $10-\mathrm{msec}$ increase in RT over compatible noise when trials were blocked. In this experiment, the difference between compatible and incompatible noise for this condition is $23 \mathrm{msec}$.

Noise compatibility has a pronounced effect for Condition 2, where the noise letters were twice as large as the target letter. In Experiment 1, on the other hand, incompatible noise had little or no effect where the size relationship of the targets and noise were the reverse. If the target is larger than the noise letters, incompatible noise interferes less with target RT than if target and noise letters are the same size. But if the situation is reversed and the target is smaller than the noise letters, then considerably more interference is obtained relative to the condition when

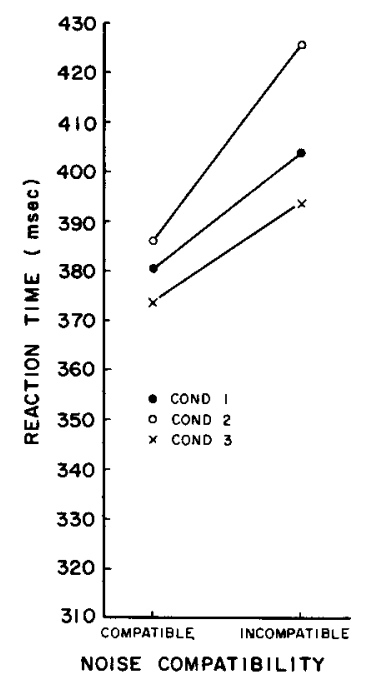

Figure 2. Mean latency for the target conditions as a function of noise compatibility. 
target and noise are the same size. This result follows predictions from the continuous flow model, and fails to support a distinctiveness explanation of the size condition data from Experiment 1.

The effect of increasing noise size relative to the target has a limit, as we anticipated that it might. In Condition 3, the noise was four times as large as the target letter. Here, the larger size of the noise has resulted in less interference in RT to the target for both compatible and incompatible noise. The difference between Conditions 1 and 3, however, is not significant $(\mathrm{p}>.05)$. We were prepared for this result due to several considerations. First of all, for a given set of forms, there is probably an optimal size range in terms of visual angle for rapid discrimination. If the size is too large, areas of the retina with less acuity become involved and a longer period of energy summation is required to compensate. Secondly, perceptual structuring factors such as grouping and figure-ground organization may play a larger role (Banks \& Prinzmetal, 1976). It was apparent to us when we first constructed the 4-to-1 ratio displays that, at the phenomenal level, the large noise letters seemed to become part of the ground from which the target letter emerged. Further exploration of optimal size and size-noise relationships is needed.

For the no-noise trials, the mean RT for the blocked trials was $328 \mathrm{msec}$ and for the mixed trials, $355 \mathrm{msec}$. The difference between these means is significant $(\mathrm{p}<.01)$ and in the direction predicted. The result is consistent with previously reported results by Eriksen and Eriksen (1974) and supports the hypothesis that subjects introduce an inhibitory stage into their behavior when they anticipate noise stimuli along with the target.

As in Experiment 1, there was no indication in the data of a speed-accuracy tradeoff. The productmoment correlation between mean RT and mean errors across the eight treatment conditions was .69 .

\section{EXPERIMENT 3}

In the previous experiments, differential effects of noise stimuli upon target RT were attributed not only to response competition but also to differences in retinal locus, size, and figure-ground contrast. We argued that the action of these latter variables was mediated via differences in visual processing time or energy summation which led to differential levels of priming in competing responses. The present experiment tested more directly the assumption of differential rate of buildup of competing responses by presenting the target and the noise stimuli asynchronously.

A backward and forward masking paradigm was employed. However, instead of a traditional mask, the target letter was preceded or followed by two response-compatible or two response-incompatible noise letters. These noise letters were so located as to flank the target position at a separation of $.5^{\circ}$ of visual angle.

The continuous flow model makes several predictions for this experiment. First, forward and backward masking will be greater when the mask consists of response-incompatible noise letters than when compatible noise letters are used as the mask. Second, in the present experimental arrangement, incompatible noise will produce more extensive forward masking than backward masking.

Little or no masking effects are predicted for response-compatible noise. Whether the noise letters precede, follow, or are simultaneous with the occurrence of the target letter, the responses that they prime are the same responses that are primed by the target, so minimum response interference occurs. This prediction is supported by previous results (Eriksen \& Eriksen, 1974; O'Hara, 1977) wherein target RT was little affected by simultaneous compatible noise when target-noise separation was great enough to eliminate contour interactions $\left(.5^{\circ}\right.$ of angle or greater).

With incompatible noise, the prediction that forward masking will be more extensive than backward masking is derived from the following considerations. In the backward arrangement, where the target precedes the incompatible noise by various stimulus onset asynchronies (SOAs), RT to the target would be greatest when the SOA was zero, and RT would decrease as the SOA increased. The function would become asymptotic at the RT level for targets presented without noise. Since priming of the noise responses cannot begin until the noise appears, delaying the noise onset results in there being a lower level of prime in competing responses at the point where the target response reaches evocation threshold. In other words, target priming has a headstart by the duration of the SOA. If the SOA is long enough, recognition or response to the target will have occurred before the noise appears.

Under the forward masking arrangement, where the noise precedes the target at various SOAs, target RT again will be unaffected by the noise stimuli if the SOA is long enough. At these longer SOAs, the noise will have resulted in recognition responses before the target occurs, and competition with the target response will not occur. As the SOAs become shorter, the level of priming for the noise responses will not have always dissipated by the time the target processing begins, resulting in increasing response competition. At very short SOAs, the competition might actually be greater than when the target and the noise are simultaneous, since the level of priming in the noise responses will be greater than that for the target.

There are two considerations that require that forward masking will be more extensive than backward. 
The first concerns the retinal locus of the noise letters relative to the target. They are on less sensitive foveal locations and, as a consequence, will require somewhat longer visual processing times for recognition responses (Eriksen \& Schultz, 1977). For example, if the retinal locus of the noise resulted in $50 \mathrm{msec}$ slower processing, then an SOA of $50 \mathrm{msec}$ between noise and target would result in equal discriminability in the developing percept.

The second consideration rests with the fact that there are two masking noise letters. It has been shown in previous research (Eriksen, 1966; Eriksen \& Lappin, 1967) that the processing and recognition of simultaneously presented letters is independent. Due to inherent variability in the nervous system, it seems safe to assume that the time required to recognize or respond to a letter is not constant. Thus, the time required to process both of two presented letters independently will average longer than for one letter alone. Consequently, the time to process both of the noise letters to the point where they do not inhibit processing the target letter will, in the forward masking paradigm, be longer than the time required to process a single target letter in the backward paradigm. The result is a longer interval of interference under forward masking.

In addition to the temporal distribution of response competitive effects, this experiment addressed a question that we raised earlier, namely, does competition occur between internal recognition responses? Experiments 1 and 2 did not provide information on this question, since the noise and target letters produced competitive effects in terms of the overt lever movement response. If competition occurred also at the covert level, the effects could not be separated from the competition occurring at the overt level.

To test for competitive effects among internal recognition responses, the present experiment used four target letters divided into two response sets, $T$ and $W$ and $A$ and $M$. Half of the subjects were instructed to move the response lever to the left if the target was an $A$ or $M$ and to the right if it was a $T$ or $W$. The instructions were reversed for the remaining subjects. This modification of the previous procedures permitted a comparison of the effects of noise when the noise and target letters were compatible on both the internal recognition response and the overt lever response (e.g., W W W) with instances where the overt response was compatible but different internal recognition responses would be activated (e.g., T W T).

\section{Method}

Subjects. Eight students of the University of Illinois, two male, served as paid volunteers. All had normal or corrected-to-normal vision, and were right-handed.

Apparatus and Stimuli. Trials were conducted on a Scientific Prototype Model GA three-field tachistoscope, illuminated with
Sylvania F4T5/CWX fluorescent bulbs. Trials were initiated at the subject's discretion by pressing a microswitch held in the left hand. Trial initiation sumultaneously onset the first tachistoscopic field and a Hunter digital clock counter set to time in milliseconds. Field luminances were set at $17.6 \mathrm{~cd} / \mathrm{m}^{2}$ in all channels, as measured by a Spectra spot photometer.

Eight stimulus cards were constructed by placing 18-point Futura Demi-bold Paratype letters (No. 11316) on vinyl cards. Four of these displays were created by placing a single capital letter $(A, M$, $\mathrm{T}$, or $\mathrm{W}$ ) on a clear vinyl card so that the letter would appear $.25^{\circ}$ of visual angle above a horizontal line (. $25^{\circ}$ of angle in length) which served as a fixation stimulus. Letters subtended $.22^{\circ}$ of visual angle in height at the 50 -in. viewing distance. The remaining four stimulus cards were constructed by placing pairs of letters (two As, two Ms, two Ts, or two Ws) on white vinyl cards, situated so as to symmetrically flank the centered target letter at a $.50^{\circ}$ intercontour distance. This intercontour distance was chosen so as to eliminate the possibilities of contour interferences (Eriksen \& Rohrbaugh, 1970, Flom, Weymouth, \& Kahneman, 1963).

When a target letter card (clear vinyl) was placed in front of a noise letter card (white vinyl) in the same tachistoscopic channel, a symmetrical three-letter display centered above the fixation line was apparent. When target and noise cards were presented in different tachistoscopic fields, a clear card was presented in front of the noise card, a white card behind the target card to maintain constant luminance in all channels. The fixation line was placed on a clear vinyl card, which was presented with a white vinyl backing card in the blank channel of the tachistoscope.

Each subject served in one practice and four experimental sessions. Four subjects were instructed to move a lever microswitch (sensitive to $1 \mathrm{~mm}$ and located under the right hand) to the left if the centered target was an $A$ or $M$ and right if it was a $T$ or $W$. The other subjects received the opposite response assignment. Each session was conducted under one level of temporal interferenceeither forward masking (flanking noise preceding the centered target) or backward masking (target preceding noise). Each session consisted of four blocks of 56 trials each. Each block of trials consisted of three replications of the 16 possible combinations of target and noise letters plus 6 trials under a no-noise control condition. Each replication of trials yielded 8 response-compatible trials (i.e., an $A$ or $M$ surrounded by As or Ms, or a $T$ or $W$ surrounded by $T s$ or Ws) and 8 response-incompatible trials (i.e., an $A$ or $M$ surrounded by Ts or Ws or a T or W surrounded by As or Ms). Thus, each block of trials yielded 24 response-compatible, 24 responseincompatible, and 6 no-noise control trials. Trial conditions were randomized within each block.

Each block of trials was conducted at a different SOA between the centered target and flanking noise letters. SOAs of $0,100,250$, and $750 \mathrm{msec}$ for forward masking and $0,50,100$, and 200 for backward masking were employed.

Trial initiation onset the first tachistoscopic field (containing the two noise letters under the forward masking condition or the centered target under the backward masking condition) while the fixation field remained on. The second stimulus field (containing the centered target for the forward masking condition or the two noise letters for the backward masking condition) onset simultaneously with offset at the fixation field at the predetermined SOA. For the 0-msec SOA blocks, the target card was placed in front of the noise card in the second tachistoscopic field and fixation would remain on for the duration of the stimulus presentation $(2,000 \mathrm{msec})$. For the asynchronous trials, the second stimulus (either target or noise) was presented for $2,000 \mathrm{msec}$, after which both the target and noise channels would offset simultaneously and the fixation line would reappear.

Trials were conducted binocularly, with latency feedback in terms of milliseconds following each trial. Five warm-up trials preceded each block, and a 5-min rest break followed the second block of trials. The subjects were instructed to respond as quickly as possible while avoiding errors, and were told that a $3 \%$ error rate was tolerable. The design yielded 96 trials per cell per subject. 


\section{Results and Discussion}

Average reaction time to targets as a function of SOA is shown in Figure 3 for the response-compatible and -incompatible noise conditions. The SOA value is the time between target and noise onset. Thus, the negative SOA values reflect the forward masking paradigm where the noise preceded the target. Also shown is the performance under the no-noise condition. A three-way ANOVA (Subjects by SOA by Noise Conditions) showed that the main effect of the noise condition was highly significant $(\mathrm{p}<.001$, df $=1,7 \mathrm{~F}=33.92$ ). Most importantly, the interaction between noise condition and SOA was significant ( $p$ $<.001$, df $=6,42, \mathrm{~F}=4.70$ ).

The data are in close agreement with our predictions. For the response-incompatible condition, where the noise letters consisted of members of the other response set, both backward and forward maskinglike functions are obtained. For the backward paradigm, where the target precedes the noise, the interference of the noise becomes progressively reduced as the SOA increases. When the target precedes the onset of the incompatible noise letters by $200 \mathrm{msec}$, RT to the target has reached the level obtained under the no-noise condition. This result indicates that, by $200 \mathrm{msec}$, target processing has progressed to the point where priming of competing responses no longer interferes.

Under the forward masking paradigm, interference is greater when the noise precedes the target by 100 msec than when they have simultaneous onset, although this difference is not significant. Target recognition latency is slower than the no-noise control level, even when the incompatible noise precedes the target by $250 \mathrm{msec}$. The continuous flow model predicted more extensive interference under the forward than under the backward paradigm. A test of this prediction is made by comparing interference at the -100 -msec SOA and at the +100 -msec SOA. A planned comparison test of this difference was significant $(\mathrm{p}<.02)$.

Consistent with previous results (Eriksen \& Eriksen, 1974; O'Hara, 1977) is the finding that response-compatible noise (noise identical to target or a member of the same response set) does not interfere with target RT when the distance between the stimuli is sufficient to avoid contour interactions (Flom, Weymouth, \& Kahneman, 1963). At all tested SOA values, target RT remained at about the no-noise control level when accompanied, preceded, or followed by response-compatible noise. In fact, there is a suggestion that target RT may have been facilitated when noise preceded it in the region of $250 \mathrm{msec}$. The reduction in target $\mathrm{RT}$ at this point does not reach significance, but it is consistent with a pathway activation effect reported by Posner and Boies (1971).

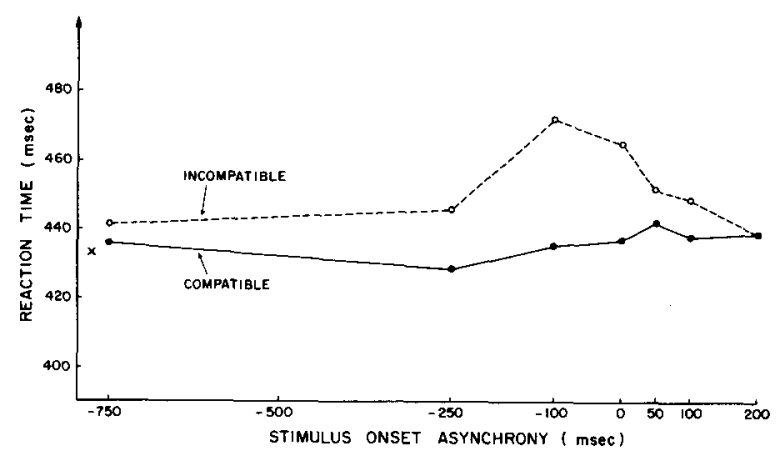

Figure 3. Mean latency to targets under compatible and incompatible noise as a function of stimulus onset asynchrony. Mean latency under the no-noise control condition, pooled over the backward-masking SOAs, is shown by the " $x$ " at the left of the figure.

While, overall, the response-compatible noise did not differ from the level of the no-noise control, in this experiment there were two forms of compatible noise: noise and target identical (e.g., W W W) and noise the other member of the same response set (e.g., T W T). In both cases, the noise and target called for the same overt lever response, but in the latter case, the subject's prior knowledge about letters would be expected to lead to a conflict in terms of an internal recognition response. To the extent that the lever movement was mediated via an internal recognition of the target letter, competition among internal recognitions would yield slower overt RTs when the noise letter was the other member of the target response set.

The analysis involved a comparison of the two kinds of compatible noise when target and noise letters were presented simultaneously $(\mathrm{SOA}=0)$. Consistent with what would be expected if internal recognition responses competed, the mean $\mathrm{RT}$ to the target was $432 \mathrm{msec}$ when the target and noise letters were identical and $443 \mathrm{msec}$ when noise was the other member in the same response set. A planned comparison of these means was significant beyond the .05 level.

There was no indication of a speed-accuracy tradeoff in this experiment. Across subjects, errors ranged from 0 to $12.5 \%$, with a mean of $6 \%$. Latencies and error rates correlated at $\mathbf{r}=.03$.

\section{GENERAL DISCUSSION}

The results of all three experiments support a visual search process that has the following characteristics: (a) Information about stimuli develops over time in the visual system; (b) several display elements are processed simultaneously and in parallel; and (c) processing of these elements continues to the incipient response activation level.

Recognition that visual information develops over time is not only consistent with the neurophysiology of the sensory system (cf. Ganz, 1975), but is assumed in 
various models of choice reaction time. The latency operating characteristic (Lappin \& Disch, 1972a, $1972 \mathrm{~b}$ ) assumes the subject's ability to respond at an earlier, less complete stage of the percept with an attendant gain in speed of response but at a cost of decreased accuracy. A similar assumption is involved in the research on time and accuracy (Pachella, 1974; Pew, 1969; Wickelgren, 1977) and the choice reaction time model of Grice, Nullmeyer, and Spiker (1977).

As we noted earlier, the essentially simultaneous processing of several elements from a display to the point of incipient response activation is quite consistent with the extensive research on the Stroop effect (see Dyer, 1973, for a review). The results of the present experiments, together with previous studies (Eriksen \& Eriksen, 1974; Keren, O’Hara, \& Skelton, 1977), make it clear that response competition or interference is more pervasive than the restrictive paradigm employed in the Stroop phenomenon. Specifically, parallel multielement processing and response competition are involved in the visual search task and most likely in all situations of multistimulus visual input.

This conclusion is difficult to integrate with discrete-stage models of visual information processing (Sternberg, 1969). The interference at the response level of extraneous or noise stimuli is inconsistent with a discrete- stage model in which the decision stage isolates earlier stage processing from the response system. A discrete-stage model might be made viable by modification of the postulated characteristics of the decision stage, but several findings of the present experiment are more consistent with a continuous flow conception. These are the variations in the interfering effects of incompatible noise that can be caused by manipulating the size, contrast, or asynchrony of noise onset. Manipulation of these variables was shown to enhance or diminish the assumed priming flow to a competing response, in a predictable manner.

The discrete-stage model introduced by Sternberg (1969) has been most influential and productive in cognitive psychology. However, alternative models that have the essential characteristics of a continuous flow conception have recently been advanced (Norman \& Bobrow, 1975; Turvey, 1973). Most recently, McClelland (Note 1) has presented a mathematical cascade model that embodies the principal characteristics of a continuous flow conception. Though none of these models specifically address response priming and competition, the cascade model can readily accommodate the data on response interference.

There are several aspects of the data in the present experiments that we feel require further comment. The first concerns the possibility of competition among internal recognition responses. While competition in terms of the overt responses is well supported, the present data do not provide clear evidence as to competition at the internal level. Eriksen and Colegate (1971) have presented evidence that the process of recognition and transfer to short-term memory of items presented in visual displays is serial in nature. If so, this would provide opportunity for competitive effects when two or more different letters were present in a display.

Results consistent with competition of internal recognition responses were obtained in Experiment 3, where it was found that RT to targets was faster when the accompanying noise was identical to the target letter than when the noise letters were the other member of the target set. In the latter case, the noise was compatible with the target in terms of the overt levermovement response but would lead to different internal recognition responses.

However, interpretation of this result is not clearcut. A difference between these two types of noise could be due to identical noise facilitating target response priming. Results from a recent experiment by Bjork and Murray (1977) do not support the possibility of identical noise facilitating target recognition, but then their findings are in conflict with several previously published experiments that found that target redundancy increased recognition accuracy at short exposure times (Eriksen, 1966; Eriksen \& Greenspon, 1968; Eriksen \& Lappin, 1965). Bjork and Murray deduced from Estes' interactive channels model (Estes, 1974) that maximum inhibition among input channels would occur when the noise in the display was identical to the target, and their results in terms of recognition accuracy supported this deduction.

Contrary results were obtained by Eriksen (1966) and Eriksen and Lappin (1965). In these experiments, identification accuracy was found to improve as a negatively accelerated function of the number of identical targets in the display. In the Eriksen and Lappin study, the number of identical targets was varied from 1 to 6 , and the results fit a model of perceptual independence which assumed that each occurrence of a target represented an independent oppor- 1 tunity to perceive. More recently, Hintzman, Carre, Eskridge, Owens, Shaff, and Sparks (1972) employed the Stroop test and found that there was a facilitation for color naming when the word was congruent with the color to be named.

It might seem that the question of facilitation or interference among internal recognition responses could be resolved by comparing the performance for "noise identical to the target" and "noise other member of the same response set" with the no-noise control in Experiment 3 of the present research. Such a comparison shows that the mean RT to targets with identical noise was $432 \mathrm{msec}$, for the no-noise control was $434 \mathrm{msec}$, and for nonidentical but same response 
set noise was $443 \mathrm{msec}$. Although latency under the identical noise condition differs significantly from that under the nonidentical same response set noise, neither of these values differs significantly from the no-noise control. Nonetheless, the ordering of the mean latencies is what would be expected if both interference and facilitation occurred. The answer, however, is not clear, and there are many methodological problems in the way of establishing definitive answers. We are not able to measure the latency of the internal recognition response directly. In the methodology of Experiment 3, conflict or facilitation among internal recognition responses would be revealed in the timing of the overt lever response only if the lever response was mediated via the internal recognition response. Such mediation may indeed be the case in early stages of practice, but it is a simple task to learn to map four letters through a direction of lever movement. When the lever-movement response and the mapping are well learned, it may well be that there is no longer mediation via the internal recognition response. Eriksen $(1956,1957)$ has previously shown that when multiple responses are required to the same stimulus, there is at least a significant amount of independence in these responses in terms of their relation to the stimulus input. Thus, in the present experiment, after initial practice, internal recognition response and the lever movement may both be independently related to the stimulus rather than recognition mediating the lever movement.

In Experiments 1 and 2, target RT was faster under the no-noise condition than it was when the target was flanked by identical noise. In Experiment 3, however, RTs were essentially the same for the no-noise and the identical noise conditions. This difference can be attributed to the spacing between target and noise that was used for the different experiments. In Experiment 1 and 2, the intercontour distance between the target and noise letters was $.25^{\circ}$ of angle, whereas in Experiment 3 this distance was $.5^{\circ}$. The closer spacing is within the range where we could anticipate contour interactions affecting the resolution of visual acuity (Flom, Weymouth, \& Kahneman, 1963). To overcome this impairment in acuity, a somewhat longer energy summation time in the visual system would be required to achieve the same degree of clarity in the percept of the target letter. Thus, less time would be required to discriminate a target presented in a blank field than would be the case where the target was surrounded at close spacing by other letters.

Contour interaction can be attributed to an effect occurring at the sensory level, but we believe that there is a second effect of spacing that occurs at distances beyond that where contour interaction occurs. The location of a form in space relative to other forms can be considered as an attribute or dimension of that stimulus or form, much as its shape, its size, or its color. Given that the subject is processing simultaneously two or more letters from the display, correct performance requires that the subject discriminate not only what letters are presented, but where they are located in order to insure that he is responding to the letter in the designated location. Mislocation or transposition errors have been shown to occur with significant frequency (Estes, Allmeyer, \& Reder, 1976). In other words, identification of a letter does not always come with precise location information. The subject is faced with essentially a conjoint discrimination, form and location. If these two attributes are processed even partially independently, and the processing time from trial to trial is variable, then it will require on the average more time to achieve a given accuracy level for both attributes than for one attribute alone.

When the space between forms or stimuli is increased, the discrimination of location should become easier. Less processing time will be required for a given level of accuracy. Eriksen and Eriksen (1974) found that target RT decreased as the spacing between target and noise was increased from .06 to $1^{8}$ of visual angle.

On the task employed in the present experiments, the subject cannot ensure correct response only by identifying the stimulus; he must also determine its location relative to the fixation point and the other letters present in the display. This requires a higher criterion before removal of inhibition for a response to occur. When the no-noise trials are run in blocks so that the subject knows there will be no accompanying noise, he can set a criterion based only upon form identification with a resultant faster reaction time. In Experiment 2, we found that blocked no-noise trials were significantly faster than no-noise trials that were intermixed with the noise conditions. Similar results were reported in the Eriksen and Eriksen (1974) study.

\section{Summary}

The reexamination of previous findings, together with new results presented here, urges the following conclusions:

(1) Stimulus evidence accumulates gradually in the visual system, over a time course of up to several hundred milliseconds.

(2) The rate of stimulus information aggregation varies as a function of such stimulus parameters as size, retinal locus, and figure-ground contrast.

(3) At stimulus onset, a broad range of responses each receive slight activation or priming. As processing continues, the range of primed responses becomes progressively more restricted.

(4) For multielement displays, primed responses for nontarget stimulus elements can inhibit the target recognition response as a function of the level of priming of these competing responses.

(5) Target discriminability from among noise or 
distractor elements can be externally manipulated by (a) placing the target on a more sensitive retinal locus than the noise, (b) giving the target higher figureground contrast than the noise, (c) increasing the target size relative to the noise, or (d) providing the target with an onset lead time relative to the noise.

\section{REFERENCE NOTE}

1. McClelland, J. L. On the time relations of mental processes: A framework for analyzing processes in cascade. Center for Human Information Processing, 1978. Report No. 77, University of California, San Diego, La Jolla, California.

\section{REFERENCES}

BAMBER, D. Reaction times and error rates for judging nominal identity of letter strings. Perception \& Psychophysics, 1972, 12, 321-326.

Banks, W. P., \& Prinzmetal, W. Configurational effects in visual information processing. Perception \& Psychophysics, 1976, 19, 361-367.

Bjork, E. L., \& MurRay, J. T. On the nature of input channels in visual processing. Psychological Review, 1977, 84, 472-484.

BRYDEN, M. P. Tachistoscopic recognition of non-alphabetical material. Canadian Journal of Psychology, 1960, 14, 78-86.

Colegate, R. L., Hoffman, J. E., \& Eriksen, C. W. Selective encoding from multielement visual displays. Perception \& Psychophysics, 1973, 14, 217-224.

Dyer, F. N. The Stroop phenomenon and its use in the'study of perceptual, cognitive, and response processes. Memory \& Cognition, 1973, 1, 106-120.

ERIKSEN, B. A., \& ERIKSEN, C. W. Effects of noise letters upon the identification of a target letter in a nonsearch task. Perception \& Psychophysics, 1974, 16, 143-149.

ERIKSEN, C. W. An experimental analysis of subception. The American Journal of Psychology, 1956, 69, 625-634.

Eriksen, C. W. Prediction from an interaction among multiple concurrent discriminative responses. Journal of Experimental Psychology, 1957, 53. 353-359.

ERIKSEN, C. W. Independence of successive inputs and uncorrelated error in visual form perception. Journal of Experimental Psychology, 1966, 72, 26-35.

Eriksen, C. W., \& Colegate, R. Selective attention and serial processing in briefly presented visual displays. Perception \& Psychophysics, 1971, 10, 321-326.

ERIKsen, C. W., \& Collins, J. F. Temporal course of selective attention. Journal of Experimental Psychology, 1969, 80, 254-261.

Eriksen, C. W., \& Greenspon, T. S. Binocular summation over time in the perception of form at brief durations. Journal of Experimental Psychology, 1968, 76, 331-336.

Eriksen, C. W., Hamlin, R. M., \& DaYe, C. The effect of flanking letters and digits on speed of identifying a letter. Bulletin of the Psychonomic Society, 1973, 2, 400-402.

Eriksen, C. W., \& Hoffman, J. E. Temporal and spatial characteristics of selective encoding from visual displays. Perception \& Psychophysics, 1972, 12, 201-204.

Eriksen, C. W., \& Hoffman, J. E. The extent of processing of noise elements during selective encoding from visual displays. Perception \& Psychophysics, 1973, 14, 155-160.

ERIKSEN, C. W., \& LAPPIN, J. S. Internal perceptual system noise and redundancy in simultaneous inputs in form identification. Psychonomic Science, 1965, 2, 351-352.

ERIKSEN, C. W., \& LAPPIN, J. S. Independence in the perception of simultaneously presented forms at brief durations. Journal of Experimental Psychology, 1967, 73, 468-472.

ERIKSEN, C. W., \& Rohrbaugh, J. Visual masking in multielement displays. Journal of Experimental Psychology, 1970, 83, 147-154.
Eriksen, C. W., \& Schultz, D. W. Retinal locus and acuity in visual information processing. Bulletin of the Psychonomic Society, 1977, 9, 81-84.

Eriksen, C. W., \& Schultz, D. W. Temporal factors in visual information processing. In J. Requin (Ed.), Attention and performance VII. New York: Academic Press, 1978.

ERIKSEN, C. W., \& Spencer, T. Rate of information processing in visual perception: Some results and methodological considerations. Journal of Experimental Psychology Monograph, 1969, 79(2, Pt. 2).

Estes, W. K. Interactions of signal and background variables in visual processing. Perception \& Psychophysics, 1972, 12, 278-286.

Estes, W. K. Redundancy of noise elements and signals in visual detection of letters. Perception \& Psychophysics, 1974, 16, 53-60.

Estes, W. K., Allmeyer, D. H., \& Reder, S. M. Serial position functions for letter identification at brief and extended exposure durations. Perception \& Psychophysics, 1976, 19, 1-15.

Estes, W. K., \& WesseL, D. L. Reaction time in relation to display size and correctness of response in forced-choice visual signal detection. Perception \& Psychophysics, 1966, 1, 369-373.

Flom, M. C., Weymouth, F. W., \& Kahneman, D. Visual resolution and contour interaction. Journal of the Optical Society of America, 1963, 53, 1026-1032.

GANZ, L. Temporal factors in visual perception. In E. C. Carterette \& M. P. Friedman (Eds.), Handbook of perception (Vol. V). New York: Academic Press, 1975.

GARDNER, G. T. Evidence for independent parallel channels in tachistoscopic perception. Cognitive Psychology, 1973, 4, 130-155.

Gatti, S. V., \& Egeth, H. E. Failure of spatial selectivity in vision. Bulletin of the Psychonomic Society, 1978, 11, 181-184.

Grice, G. R., Nullmeyer, R., \& Spiker, V. A. Application of variable criterion theory to choice reaction time. Perception \& Psychophysics, 1977, 22, 431-449.

Hintzman, D. L., Carree, F. A., Eskridge, V. L., Owens, A. M., Shaff, S. S., \& Sparks, M. E. "Stroop" effect: Input or output phenomenon? Journal of Experimental Psychology, $1972,95,458-459$.

Kahneman, D. Attention and effort. Englewood Cliffs, N.J: Prentice-Hall, 1973.

KahNEman, D., \& NoRman, J. The time-intensity relation in visual perception as a function of the observer's task. Journal of Experimental Psychology, 1964, 68, 215-220.

Kahneman, D., Norman, J., \& Kubovy, M. The critical duration for the resolution of form: Centrally or peripherally determined? Journal of Experimental Psychology, 1967, 73, 323-327.

Keren, G., O'Hara, W. P., \& Skelton, J. M. Levels of noise processing and attentional control. Joumal of Experimental Psychology: Human Perception and Performance, 1977, 3, 653-664.

KinChlA, R. A. Detecting target elements in multielement arrays. Perception \& Psychophysics, 1974, 15, 149-158.

LAPPIN, J. S., \& Disch, K. The latency operating characteristic: I. Effects of stimulus probability on choice reaction time. Journal of Experimental Psychology, 1972, 92, 419-427. (a)

LAPPIN, J. S., \& DisCH, K. The latency operating characteristic: II. Effects of visual stimulus intensity on choice reaction time. Journal of Experimental Psychology, 1972, 93, 367-372. (b)

LEFTON, L. A., \& HABER, R. N. Information extraction from different retinal locations. Journal of Experimental Psychology, 1974, 102, 975-980.

MEWHORT, D. J. K. Accuracy and order of report in tachistoscopic identification. Canadian Journal of Psychology, 1974, 28, 383-398.

NeILL, W. T. Decision processes in selective attention: Response priming in the Stroop color-word task. Perception \& Psychophysics, 1978, 23, 80-84.

NeIsser, U. Cognitive psychology. New York: Appleton-CenturyCrofts, 1967. 
Norman, D. A., \& Bobrow, D. G. On data limited and resource limited processes. Cognitive Psychology, 1975, 7, 44-64.

O'HARA, W. P. The spatial and featural effects of irrelevant letters on the speed of same-different judgments of two target letters. Master's thesis, University of Illinois, UrbanaChampaign, 1977.

Pachella, R. The interpretation of reaction time in information processing research. In B. Kantowitz (Ed.), Human information processing: Tutorials in performance and cognition. New York: Halsted Press, 1974.

PEw, R. W. The speed-accuracy operating characteristic. In W. C. Koster (Ed.), Attention and performance II. Amsterdam: North-Holland, 1969. (Reprinted from Acta Psychologica, 1969, 30, 16-26.)

Posner, M. I., \& Boies, S. J. Components of attention. Psychological Review, 1971, 78, 391-408.

Prinzmetal, W., \& Banks, W. P. Good continuation affects visual detection. Perception \& Psychophysics, 1977, 21, 389-395.

Proctor, R. W. Sources of color-word interference in the Stroop color-naming task. Perception \& Psychophysics, 1978, 23, 413-419.

Schultz, D. W., \& Eriksen, C. W. Stimulus size and acuity in visual information processing. Bulletin of the Psychonomic Society, 1978, 12, 397-399.

Shaw, P., \& Weigel, G. A. Effects of bars and blanks on recognition of words and non-words embedded in a row of letters. Perception \& Psychophysics, 1973, 14, 117-124.

ShIfFrin, R. M., \& GeisLer, W. A. Visual recognition in a theory of information processing. In R. L. Solso (Ed.), Contemporary issues in cognitive psychology: The Loyola symposium. Washington, D.C: Winston, 1973.
STERnberg, S. Memory scanning: Mental processes revealed by reaction time experiments. American Scientist, 1969, 57, 421-457.

STRANGert, B., \& BRÄnNström, L. Spatial interaction effects in letter processing. Perception \& Psychophysics, 1975, 17, 268-272.

Townsend, J. T., TAYLOR, S. G., \& BRown, D. R. Lateral masking for letters with unlimited viewing time. Perception \& Psychophysics, 1971, 10, 375-378.

TURVEY, M. On peripheral and central processes in vision: Inferences from an information-processing analysis of masking with patterned stimuli. Psychological Review, 1973, 80, 1-52.

VoN WRIGHT, J. M. Selection in visual immediate memory. Quarterly Journal of Experimental Psychology, 1968, 20, 62-68.

VON WRIGHT, J. M. On selection in immediate visual memory. In A. F. Sanders (Ed.), Attention and performance III. Amsterdam: North Holland, 1970.

Wickelgren, W. A. Speed-accuracy tradeoff and information processing dynamics. Acta Psychologica, 1977, 41, 67-85.

Wolford, G., \& Hollingsworth, S. Retinal location and string position as important variables in visual information processing. Perception \& Psychophysics, 1974, 16, 437.442.

Wolford, G. L., Wessel, D. L., \& Estes, W. K. Further evidence concerning scanning and sampling assumptions of visual detection models. Perception \& Psychophysics, 1968, 3, 439.444.

(Received for publication September 6, 1978; revision accepted January $23,1979$. 